\title{
Association between Helicobacter pylori Infection and Nonalcoholic Fatty Liver Disease: A Single-Center Clinical Study
}

\author{
Ou Cai $\mathbb{D}^{1},{ }^{1}$ Zhenpeng Huang, ${ }^{2}$ Ming Li $\mathbb{D},{ }^{1}$ Chaoqun Zhang $\left(\mathbb{D},{ }^{1}\right.$ Fengbo Xi, ${ }^{3}$ \\ and Shiyun Tan (1) ${ }^{1}$ \\ ${ }^{1}$ Department of Gastroenterology, Renmin Hospital of Wuhan University, Wuhan, Hubei, China \\ ${ }^{2}$ Teaching and Research Section of Internal Medicine, College of Clinical Medicine, Xi'an Medical University, Xi'an, Shannxi, China \\ ${ }^{3}$ Enshi Prefecture Center for Disease Control and Prevention, Enshi, China
}

Correspondence should be addressed to Shiyun Tan; tanshiyun@medmail.com.cn

Received 2 September 2017; Revised 11 November 2017; Accepted 23 November 2017; Published 21 January 2018

Academic Editor: Francesco Selvaggi

Copyright ( $) 2018$ Ou Cai et al. This is an open access article distributed under the Creative Commons Attribution License, which permits unrestricted use, distribution, and reproduction in any medium, provided the original work is properly cited.

Objective. To investigate the association between Helicobacter pylori (H. pylori) infection and nonalcoholic fatty liver disease (NAFLD). Methods. Data from 2051 participants who underwent ${ }^{13} \mathrm{C}$ urea breath test and abdominal ultrasound examinations was collected. Participants were allocated to NAFLD risk group and NAFLD nonrisk group based on definite risk factors for NAFLD. The relationship between $H$. pylori infection and NAFLD was analyzed. Results. No significant difference was found between rates of $H$. pylori infection and NAFLD using the chi-square test $(P=0.30)$ or regression analysis $(P=0.70)$. There was no significant difference between rates of $H$. pylori infection with and without NAFLD $(P=0.47)$ in the NAFLD risk group or in the NAFLD nonrisk group $(P=0.59)$. There was no significant difference between rates of $H$. pylori infection in men $(P=0.69)$ and in women $(P=0.27)$ or in participants aged 18-40 years $(P=0.43), 41-65$ years $(P=0.14)$, and $\geq 66$ years $(P=0.66)$ with and without NAFLD in the NAFLD risk group or between the same sex or age groups $(P=0.82, P=0.66, P=0.24, P=0.53$, and $P=1.00$, resp.) in the NAFLD nonrisk group. Conclusions. H. pylori infection does not appear to increase the NAFLD prevalence rate or to be associated with, or a risk factor for, NAFLD.

\section{Introduction}

Nonalcoholic fatty liver disease (NAFLD) is a type of liver injury induced by metabolic stress and is related to insulin resistance (IR) and hereditary susceptibility [1]. It is generally deemed to be the hepatic manifestation of metabolic syndrome [2]. NAFLD comprises simple nonalcoholic fatty liver (NAFL), nonalcoholic steatohepatitis (NASH) and NASH-associated liver cirrhosis, and hepatocellular carcinoma [1]. The incidence of and morbidity from NAFLD have increased rapidly worldwide, with corresponding increases in clinical and economic burden [3]. Apart from known and common risk factors, such as obesity, type 2 diabetes, hypertension, and dyslipidemia, it has recently been postulated that Helicobacter pylori infection is involved in the pathogenesis of insulin resistance (IR) $[1,4]$ and may be associated with NAFLD.

H. pylori is a Gram-negative bacterium which selectively colonizes the gastric mucosa [5] and is considered to be the main pathogenic bacteria involved in peptic ulcers, chronic active gastritis, mucosa-associated lymphoid tissue lymphoma, and gastric cancer [6-8]. Recently, H. pylori infection has been implicated in various nongastrointestinal diseases including idiopathic thrombocytopenic purpura, cardiovascular disease, type II diabetes, and iron deficiency anemia [9-11]. However, the findings of recent studies focusing on the relationship between $H$. pylori infection and NAFLD 
Participants who underwent abdominal ultrasound and ${ }^{13} \mathrm{C}$ urea breath test as part of routine health examination from June to December of $2016(n=2985)$

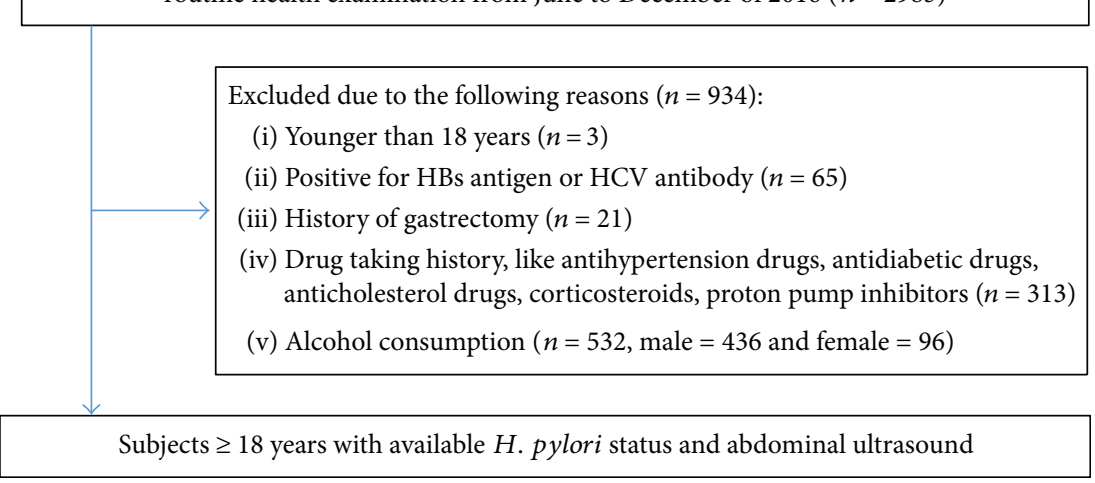

FIGURE 1: Of the 2985 participants attended, 2051 participants were analyzed in our study.

TABLE 1: Definition of $H$. pylori infection, NAFLD, and NAFLD risk group.

\begin{tabular}{clcc}
\hline & Item & Value & Standard \\
\hline H. pylori infection & ${ }^{13} \mathrm{C}$ urea breath test & $<$ delta $>\%$ (30 minutes) minus $<$ delta $>\%$ (0 minutes) & Positive: $\geq 4$ \\
& & Negative: $<4$ & \\
\hline
\end{tabular}

(i) The near-field echo of the liver is diffusely increased and more than the kidney.

NAFLD Abdominal ultrasound (ii) The intrahepatic duct structure is not clear.

Positive: any two of the three satisfied

(iii) The far-field echo of the liver is decreased Negative: none or only one satisfied gradually.

\begin{tabular}{|c|c|c|c|}
\hline \multirow{8}{*}{ NAFLD risk or not } & \multirow{4}{*}{ Blood lipid } & $\mathrm{TG} \geq 1.70 \mathrm{mmol} / \mathrm{L}$ & \multirow{8}{*}{$\begin{array}{l}\text { Risk group: one or more of the eight } \\
\text { satisfied } \\
\text { Nonrisk group: none of the eight } \\
\text { satisfied }\end{array}$} \\
\hline & & $\mathrm{TC} \geq 5.20 \mathrm{mmol} / \mathrm{L}$ & \\
\hline & & $\mathrm{HDL}-\mathrm{C}<1.00 \mathrm{mmol} / \mathrm{L}$ & \\
\hline & & $\mathrm{LDL}-\mathrm{C} \geq 3.10 \mathrm{mmol} / \mathrm{L}$ & \\
\hline & Fast plasma glucose & $\mathrm{FPG} \geq 6.10 \mathrm{mmol} / \mathrm{L}$ & \\
\hline & & $\mathrm{SBP} \geq 140 \mathrm{mmHg}$ & \\
\hline & Blood pressure & $\mathrm{DBP} \geq 90 \mathrm{mmHg}$ & \\
\hline & Body mass index & $\mathrm{BMI} \geq 24.90 \mathrm{~kg} / \mathrm{m}^{2}$ & \\
\hline
\end{tabular}

NAFLD: nonalcoholic fatty liver disease; TG: triglyceride; TC: total cholesterol; HDL-C: high-density lipoprotein cholesterol; LDL-C: low-density lipoprotein cholesterol; FPG: fast plasma glucose; SBP: systolic blood pressure; DBP: diastolic blood pressure; BMI: body mass index.

are inconsistent [12-15]. Our study used data from volunteers who underwent physical examinations in the Renmin Hospital of Wuhan University from June to December 2016 in order to analyze the association between $H$. pylori infection and NAFLD prevalence rates, to elucidate this relationship, and to provide a new strategy for treatment of NAFLD.

\section{Materials and Methods}

2.1. Study Subjects. It was a cross-sectional study of Chinese asymptomatic adults who underwent physical examination in the Renmin Hospital of Wuhan University from June 2016 to December 2016 and entered into our study voluntarily (Figure 1). This study gained approval by the ethics committees of the Renmin Hospital of Wuhan University. We included participants who underwent ${ }^{13} \mathrm{C}$ urea breath test and abdominal ultrasound $(n=2985)$. We excluded 934 subjects due to the following reasons: younger than
18 years old $(n=3)$; positive for $\mathrm{HBs}$ antigen or $\mathrm{HCV}$ antibody $(n=65)$; history of gastrectomy $(n=21)$; drug taking history, such as antihypertension drugs, antidiabetic drugs, anticholesterol drugs, corticosteroids, and proton pump inhibitors $(n=313)$; and alcohol consumption more than $140 \mathrm{~g} /$ week for male and $70 \mathrm{~g} /$ week for female $(n=532$, male $=436$ and female $=96)$. 2051 participants older than 18 years with available $H$. pylori status and abdominal ultrasound were finally analyzed. All participants were divided into NAFLD risk group and NAFLD nonrisk group according to definite NAFLD risk factors, including dyslipidemia (TG $\geq 1.70 \mathrm{mmol} / \mathrm{L}, \mathrm{TC} \geq 5.20 \mathrm{mmol} / \mathrm{L}$, HDL$\mathrm{C}<1.00 \mathrm{mmol} / \mathrm{L}$, and $\mathrm{LDL}-\mathrm{C} \geq 3.10 \mathrm{mmol} / \mathrm{L})$, high blood glucose $(F P G \geq 6.10 \mathrm{mmol} / \mathrm{L})$, high blood pressure $(\mathrm{SBP} \geq$ $140 \mathrm{mmHg}$ and $\mathrm{DBP} \geq 90 \mathrm{mmHg}$ ), and $\mathrm{BMI} \geq 24.90 \mathrm{~kg} / \mathrm{m}^{2}$. Participants with any one or more of the eight items are included in the NAFLD risk group, and those with none of the eight are included in the NAFLD nonrisk group (Table 1). 
TABLE 2: Baseline characteristics of study participants.

\begin{tabular}{|c|c|c|c|c|c|}
\hline & All $(n=2051)$ & NAFLD $(-)(n=1618)$ & NAFLD $(+)(n=433)$ & Statistics & $P$ value \\
\hline Age (y) & $38.11 \pm 10.49$ & $37.25 \pm 10.38$ & $41.31 \pm 10.30$ & $t=-7.24$ & 0.00 \\
\hline Sex (male/female) & $714 / 1337$ & $446 / 1172$ & $268 / 165$ & $\chi^{2}=177.39$ & 0.00 \\
\hline BMI $\left(\mathrm{kg} / \mathrm{m}^{2}\right)$ & $23.45 \pm 3.05$ & $22.63 \pm 2.55$ & $26.52 \pm 2.77$ & $t=-27.66$ & 0.00 \\
\hline $\mathrm{SBP}(\mathrm{mmHg})$ & $118.30 \pm 14.52$ & $116.37 \pm 13.71$ & $125.53 \pm 15.18$ & $t=-12.07$ & 0.00 \\
\hline $\mathrm{DBP}(\mathrm{mmHg})$ & $71.11 \pm 10.07$ & $69.77 \pm 9.62$ & $76.11 \pm 10.18$ & $t=-12.03$ & 0.00 \\
\hline $\mathrm{TG}(\mathrm{mmol} / \mathrm{L})$ & $1.29 \pm 1.15$ & $1.06 \pm 0.66$ & $2.18 \pm 1.91$ & $t=-12.01$ & 0.00 \\
\hline $\mathrm{TC}(\mathrm{mmol} / \mathrm{L})$ & $4.43 \pm 0.80$ & $4.35 \pm 0.77$ & $4.73 \pm 0.85$ & $t=-8.46$ & 0.00 \\
\hline HDL-C (mmol/L) & $1.34 \pm 0.36$ & $1.40 \pm 0.36$ & $1.11 \pm 0.24$ & $t=19.29$ & 0.00 \\
\hline LDL-C (mmol/L) & $2.44 \pm 0.67$ & $2.37 \pm 0.64$ & $2.73 \pm 0.70$ & $t=-10.14$ & 0.00 \\
\hline $\mathrm{FPG}(\mathrm{mmol} / \mathrm{L})$ & $5.19 \pm 0.98$ & $5.07 \pm 0.56$ & $5.66 \pm 1.76$ & $t=-6.96$ & 0.00 \\
\hline H. pylori infection $(-/+)$ & $1406 / 645$ & $1118 / 500$ & $288 / 145$ & $\chi^{2}=1.06$ & 0.30 \\
\hline
\end{tabular}

Values are expressed as means \pm standard deviation. NAFLD: nonalcoholic fatty liver disease; BMI: body mass index; SBP: systolic blood pressure; DBP: diastolic blood pressure; TG: triglyceride; TC: total cholesterol; HDL-C: high-density lipoprotein cholesterol; LDL-C: low-density lipoprotein cholesterol; FPG: fast plasma glucose.

2.2. General Information. We used uniformly trained nurses to enquire and fill in the uniformly designed epidemiologic questionnaires. In the morning, we routinely examined the height, weight, and blood pressure of every participant. The body mass index (BMI) was the weight $(\mathrm{kg})$ divided by the square of height in meters.

2.3. Serum Biochemical Examination. Morning fasting venous blood of all participants was obtained to detect the levels of serum triglyceride (TG), cholesterol (TC), high-density lipoprotein cholesterol (HDL-C), low-density lipoprotein cholesterol (LDL-C), and fasting plasma glucose (FPG) with Backman AU5800 biochemical analyzer (Backman Coulter Commercial Enterprise (China) Co. Ltd., Beijing, China).

2.4. H. pylori Infection Test. The urea $\left({ }^{13} \mathrm{C}\right)$ capsule breath test box (Shenzhen Zhonghe Headway Bio-Sci \& Tech Co. Ltd., Shenzhen, Guangdong, China, Batch number 0520150904) and HUBT-20A breath test tester (Shenzhen Zhonghe Headway Bio-Sci \& Tech Co. Ltd., Shenzhen, Guangdong, China) were used for detection. The procedure was as follows: (i) fill in the information of the participant in the two prepared collection bags; (ii) collect the air exhaled by the participant in quiet condition in one collection bag as the 0 minute sample; (iii) collect the air again after taking the urea $\left({ }^{13} \mathrm{C}\right)$ capsule with 80 to 100 milliliter water for 30 minutes as the 30 minutes sample; (iv) detect the sample in 0 and 30 minutes in the HUBT-20A breath test tester to get the corresponding value; (v) use $<$ delta $>\%$ to express the result and detection value equals $<$ delta $>\%$ (30 minutes) minus $<$ delta $>\%$ o ( 0 minutes). When detection value was no less than 4.0, we regarded it as positive and according to the Fourth Chinese National Consensus Report on the management of Helicobacter pylori infection [16] (Table 1), the participant was in current infection.
2.5. Ultrasonic Measurement. Philips iE Elite and iE 33 (Philips China Investment Co., Beijing, China) were used for abdominal ultrasound by professional ultrasound doctors. According to the guidelines for the diagnosis and management of nonalcoholic fatty liver disease: update 2010 [1], participants who possessed two of the following three characteristics could be diagnosed as fatty liver: (i) the near-field echo of the liver is diffusely increased and more than the kidney; (ii) the intrahepatic duct structure is not clear; (iii) the far-field echo of the liver is decreased gradually (Table 1). Three professional ultrasound doctors were uniformly trained for the diagnosis of fatty liver, and each participant was measured by two of them. The diagnosis could not be made until at least two of the doctors made an agreement.

2.6. Statistical Analysis. Software SPSS version 18.0 was used for statistical analysis. Continuous data accorded with normal distribution were presented as average \pm standard deviation, and categorical data were presented as number (percentage). Continuous variables were compared by $t$-test, and categorical variables were compared by chi-square test. Regression analysis was used to identify independent risk factors. $P<0.05$ was regarded as indicating statistical significance.

\section{Results}

3.1. Baseline Characteristics. Baseline characteristics of the participants are shown in Table 2. The mean age was $38.11 \pm 10.49$ years, and the prevalence of NAFLD was $21.11 \%(433 / 2051)$. Compared to participants without NAFLD, those with NAFLD were more likely to be older and male, with higher BMI, SBP, and DBP, higher levels of TG, TC, LDL-C, and FPG, and lower levels of HDL-C $(P=0.00)$. However, there was no association between $H$. pylori infection and NAFLD $(P=0.30)$ (Table 2). 
TABLE 3: Independent risk factors for NAFLD by regression analysis.

\begin{tabular}{lccccc}
\hline & $B$ & SE & Wald & Exp $(B)$ & $95 \%$ CI \\
\hline Sex & -0.40 & 0.15 & 6.83 & 0.69 & $0.49-0.90$ \\
BMI & 0.39 & 0.03 & 163.17 & 1.47 & $1.39-1.56$ \\
TG & 0.55 & 0.11 & 26.31 & 1.73 & $1.40-2.14$ \\
HDL-C & -1.32 & 0.38 & 12.19 & 0.27 & 0.01 \\
FPG & 0.42 & 0.10 & 16.14 & 1.52 & $0.13-0.56$ \\
H. pylori infection & -0.06 & 0.15 & 0.15 & 0.94 & 0.00 \\
\hline
\end{tabular}

BMI: body mass index; TG: triglyceride; HDL-C: high-density lipoprotein cholesterol; FPG: fast plasma glucose.

TABLe 4: Association analysis between $H$. pylori infection and NAFLD.

\begin{tabular}{|c|c|c|c|c|c|c|}
\hline & \multicolumn{3}{|c|}{ NAFLD risk group $(n=1072)$} & \multicolumn{3}{|c|}{ NAFLD nonrisk group $(n=979)$} \\
\hline & Positive & Negative & $\chi^{2}(P)$ & Positive & Negative & $\chi^{2}(P)$ \\
\hline Whole participate & & & $0.51(0.47)$ & & & $0.38(0.59)$ \\
\hline H. pylori $(+)$ & 137 (38.92\%) & $215(61.18 \%)$ & & $8(2.73 \%)$ & $285(97.27 \%)$ & \\
\hline H. pylori $(-)$ & $264(36.67 \%)$ & $456(63.33 \%)$ & & $24(3.50 \%)$ & $662(96.50 \%)$ & \\
\hline \multicolumn{7}{|l|}{ In different sex } \\
\hline Male & & & $0.16(0.69)$ & & & $0.05(0.82)$ \\
\hline H. pylori $(+)$ & $81(47.65 \%)$ & $89(52.35 \%)$ & & $3(5.36 \%)$ & $53(94.64 \%)$ & \\
\hline H. pylori $(-)$ & $176(45.83 \%)$ & $208(54.17 \%)$ & & $8(7.69 \%)$ & $96(92.31 \%)$ & \\
\hline Female & & & $1.23(0.27)$ & & & $0.28(0.60)$ \\
\hline H. pylori $(+)$ & $56(30.77 \%)$ & $126(69.23 \%)$ & & $5(2.11 \%)$ & $232(97.89 \%)$ & \\
\hline H. pylori $(-)$ & $88(26.19 \%)$ & $248(73.81 \%)$ & & $16(2.75 \%)$ & $566(97.25 \%)$ & \\
\hline \multicolumn{7}{|l|}{ In different age } \\
\hline $18-40$ & & & $0.62(0.43)$ & & & $1.36(0.24)$ \\
\hline H. pylori $(+)$ & $54(32.53 \%)$ & $112(67.47 \%)$ & & $3(1.50 \%)$ & $197(98.50 \%)$ & \\
\hline H. pylori $(-)$ & $138(36.03 \%)$ & $245(63.97 \%)$ & & $18(3.48 \%)$ & $499(96.52 \%)$ & \\
\hline $41-65$ & & & $2.16(0.14)$ & & & $0.40(0.53)$ \\
\hline H. pylori $(+)$ & $79(44.63 \%)$ & $98(55.37 \%)$ & & $5(5.43 \%)$ & 87 (94.57\%) & \\
\hline H. pylori $(-)$ & $122(37.89 \%)$ & $200(62.11 \%)$ & & $5(3.01 \%)$ & $161(96.99 \%)$ & \\
\hline$\geq 66$ & & & $0.20(0.66)$ & & & $-(1.00)$ \\
\hline H. pylori $(+)$ & $4(44.44 \%)$ & $5(55.56 \%)$ & & $0(0)$ & $1(100 \%)$ & \\
\hline H. pylori $(-)$ & $4(26.67 \%)$ & $11(73.33 \%)$ & & $1(33.33 \%)$ & $2(66.67 \%)$ & \\
\hline
\end{tabular}

3.2. Risk Factors for NAFLD by Regression Analysis. Regression analysis was used to analyze the independent risk factors for NAFLD, and all the factors which were significant according to $t$-tests or chi-square tests were included, plus $H$. pylori infection. Analysis showed that sex $(P=0.01)$ and BMI and levels of TG, HDL-C, and FPG were independent risk factors for NAFLD $(P=0.00)$ but $H$. pylori infection was not $(P=0.70)($ Table 3$)$.

3.3. Association between $H$. pylori Infection and NAFLD. In the NAFLD risk group, the $H$. pylori infection rate among participants with NAFLD (34.16\%) was slightly higher than that among participants without NAFLD (32.04\%), although the difference was not significant $(P=0.47)$. There was also no significant difference between the rates of $H$. pylori infection and NAFLD in men $(P=0.69)$ and women $(P=0.27)$. Moreover, after age stratification, no significant difference was found between the rates of $H$. pylori infection and
NAFLD in younger $(18-40$ years $)(P=0.43)$, middle-aged (41-65 years) $(P=0.14)$, and older $(\geq 66$ years) participants $(P=0.66)$. In the NAFLD nonrisk group, the $H$. pylori infection rate among participants with NAFLD (25.00\%) was lower than that among participants without NAFLD $(30.10 \%)$, although the results were not significant $(P=0.59)$. Similar results were also obtained for men $(P=0.82)$, women $(P=0.60)$, younger $(P=0.24)$, middle-aged $(P=0.53)$, and older participants $(P=1.000)$ (Table 4$)$.

\section{Discussion}

NAFLD is a common metabolic disorder that affects approximately $13.48 \%-31.79 \%$ of the general population [3], although its mechanism remains unclear. Genetic, environmental, and metabolic factors may be involved in the pathogenesis of NAFLD, and some recent research provides insights into the link between $H$. pylori infection 
and NAFLD. In 2008, Cindoruk et al. [17] found $H$. pylori $16 \mathrm{~S}$ rDNA in a biopsy taken from a 44-year-old woman with NASH and, in 2009, Pirouz et al. [18] found $5 \mathrm{H}$. pylori $16 \mathrm{~S}$ rRNA-positive patients among 11 biopsy-proven NAFLD patients, compared with 2 positive patients among 13 controls. This indicated that $H$. pylori could infect the liver and aroused academic interest. However, it has been difficult to culture H. pylori from the liver [19], suggesting that it cannot successfully colonize the liver and is therefore unlikely to cause damage.

Research into the mechanism underlying the relationship between $H$. pylori infection and NAFLD has mainly focused on IR. In 2011, a systematic review conducted by Polyzos et al. [4] concluded that there was a positive association between $H$. pylori infection and IR but significant heterogeneity had been found between studies and further research was therefore needed. In 2013, Abenavoli et al. [20] conducted a study that found that serum insulin levels and homeostatic model assessment- (HOMA-) IR were lower after $H$. pylori eradication and concluded that $H$. pylori eradication might improve IR. However, the intrinsic mechanism still remained obscure. In 2013, Polyzos et al. [12] first showed that liver biopsy-proven NAFLD patients had significantly higher anti- $H$. pylori IgG levels and demonstrated that $H$. pylori infection might contribute to NAFLD directly or indirectly via IR, since NAFLD is an independent predictor of IR. However, this study included a relatively small number of participants (28), and $H$. pylori seropositivity, which cannot distinguish between current and past infection, was used to detect $H$. pylori infections. All these studies indicate that there is a positive association between $H$. pylori infection and IR but have not identified a definite pathophysiological mechanism.

No association between $H$. pylori infection and NAFLD was found in two recent large clinical trials. Okushin et al. [13] conducted a large-scale study in Japan in 2015 of 13,737 participants. The authors concluded that BMI, platelet count, and serum alanine aminotransferase levels were associated with NAFLD but $H$. pylori infection was not. In 2016, Baeg et al. [14] analyzed data from 3663 patients and concluded that smoking and C-reactive protein concentration were risk factors for NAFLD but $H$. pylori infection was not. Our study also found that $H$. pylori infection was not associated with NAFLD, even after stratifying by sex and age.

Our study has some limitations. The first is that as a single-center cross-sectional study, it was difficult to infer causation and our study therefore represents a low level of evidence. The second is the use of abdominal ultrasound to diagnose NAFLD, since the results are operator dependent. However, we attempted to mitigate error by ensuring that the doctors in our study were trained according to the same protocol and a confirmed diagnosis was based on the opinion of at least two doctors. A third limitation is that we did not grade the severity of NAFLD or distinguish NASH from NAFL. Sumida et al. [15] found that the prevalence of NASH and the grade of hepatocyte ballooning were higher in $H$. pylori-seropositive patients, indicating that $H$. pylori infection may act as a contributing factor in the progression of
NAFL to NASH but not in early-stage NAFLD. In spite of its limitations, our study has some advantages. To our knowledge, ours is the first study to group data by definite risk factors for NAFLD into an NAFLD risk group and NAFLD nonrisk group. We also excluded the effect of age and sex by analyzing and stratifying the data and found a similar trend of no association between the stratified data; this strengthens our conclusions.

To conclude, although, to date, the association between H. pylori infection and NAFLD has been controversial and our research found no association despite limitations in the design of our study. Our large cross-sectional study focused on the relationship between $H$. pylori infection and NAFLD. We found that $H$. pylori infection did not associate with NAFLD, even when the data were stratified according to sex and age. Our findings therefore indicate that $H$. pylori infection has no association with NAFLD. Further largescale multicenter prospective studies are urgently needed to investigate whether there is any association between $H$. pylori infection and NAFLD and to clarify the intrinsic mechanisms involved.

\section{Conflicts of Interest}

The authors declare that they have no conflicts of interest.

\section{References}

[1] J. G. Fan, J. D. Jia, Y. M. Li et al., "Guidelines for the diagnosis and management of nonalcoholic fatty liver disease: update 2010," Journal of Digestive Diseases, vol. 12, no. 1, pp. 3844, 2011.

[2] Y. Yilmaz, "NAFLD in the absence of metabolic syndrome: different epidemiology, pathogenetic mechanisms, risk factors for disease progression?," Seminars in Liver Disease, vol. 32, no. 01, pp. 014-021, 2012.

[3] Z. M. Younossi, A. B. Koenig, D. Abdelatif, Y. Fazel, L. Henry, and M. Wymer, "Global epidemiology of nonalcoholic fatty liver disease-meta-analysis assessment of prevalence, incidence and outcomes," Hepatology, vol. 64, no. 1, pp. 7384, 2016.

[4] S. A. Polyzos, J. Kountouras, C. Zavos, and G. Deretzi, "The association between Helicobacter pylori infection and insulin resistance: a systemic review," Helicobacter, vol. 16, no. 2, pp. 79-88, 2011.

[5] P. Malfertheiner, A. Link, and M. Selgrad, "Helicobacter pylori: perspectives and time trends," Nature Reviews. Gastroenterology \& Hepatology, vol. 11, no. 10, pp. 628-638, 2014.

[6] B. Marshall and J. R. Warren, "Unidentified curved bacilli in the stomach of patients with gastritis and peptic ulceration," Lancet, vol. 323, no. 8390, pp. 1311-1315, 1984.

[7] R. Pellicano, D. G. Ribaldone, S. Fagoonee, M. Astegiano, G. M. Saracco, and F. Mégraud, "A 2016 panorama of Helicobacter pylori infection: key messages for clinicans," Panminerva Medica, vol. 58, no. 4, pp. 304-317, 2016.

[8] P. Malfertheiner, F. K. Chan, and K. E. McColl, "Peptic ulcer disease," Lancet, vol. 374, no. 9699, pp. 1449-1461, 2009.

[9] P. Malfertheiner, F. Megraud, C. A. O'Morain et al., "Management of Helicobacter pylori infection-the Maastricht V/ Florence Consensus Report," Gut, vol. 66, no. 1, pp. 6-30, 2017. 
[10] F. Franceschi, A. Gasbarrini, S. A. Polyzos, and J. Kountouras, "Extragastric diseases and Helicobacter pylori," Helicobacter, vol. 20, Supplement 1, pp. 40-46, 2015.

[11] W. A. Nasif, M. H. Mukhtar, M. M. Nour Eldein, and S. S. Ashgar, "Oxidative DNA damage and oxidized low density lipoprotein in type II diabetes mellitus among patients with Helicobacter pylori infection," Diabetology and Metabolic Syndrome, vol. 8, no. 1, p. 34, 2016.

[12] S. A. Polyzos, J. Kountouras, A. Papatheodorou et al., "Helicobacter pylori infection in patients with nonalcoholic fatty liver disease," Metabolism, vol. 62, no. 1, pp. 121-126, 2013.

[13] K. Okushin, Y. Takahashi, N. Yamamichi et al., "Helicobacter pylori infection is not associated with fatty liver disease including non-alcoholic fatty liver disease: a large-scale cross-sectional study in Japan," BMC Gastroenterology, vol. 15, no. 1, p. 25, 2015.

[14] M. K. Baeg, S. K. Yoon, S. H. Ko, Y. S. Noh, I. S. Lee, and M. G. Choi, "Helicobacter pylori infection is not associated with nonalcoholic fatty liver disease," World Journal of Gastroenterology, vol. 22, no. 8, pp. 2592-2600, 2016.

[15] Y. Sumida, K. Kanemasa, S. Imai et al., "Helicobacter pylori infection might have a potential role in hepatocyte ballooning in nonalcoholic fatty liver disease," Journal of Gastroenterology, vol. 50, no. 9, pp. 996-1004, 2015.

[16] Chinese Society of Gastroenterology, Chinese study group on helicobacter pylori, W. Z. Liu, Y. Xie et al., "Fourth Chinese National Consensus Report on management of Helicobacter pylori infection," Journal of Digestive Diseases, vol. 14, no. 5, pp. 211-221, 2013.

[17] M. Cindoruk, M. Y. Cirak, S. Unal et al., "Identification of helicobacter species by $16 \mathrm{~S}$ rDNA PCR and sequence analysis in human liver samples from patients with various etiologies of benign liver diseases," European Journal of Gastroenterology \& Hepatology, vol. 20, no. 1, pp. 33-36, 2008.

[18] T. Pirouz, L. Zounubi, H. Keivani, N. Rakhshani, and M. Hormazdi, "Detection of Helicobacter pylori in paraffinembedded species from patients with chronic liver diseases, using the amplification method," Digestive Diseases and Sciences, vol. 54, no. 7, pp. 1456-1459, 2009.

[19] R. Pellicano, A. Menard, M. Rizzetto, and F. Mégraud, "Helicobacter species and liver diseases: association or causation?," The Lancet Infectious Diseases, vol. 8, no. 4, pp. 254260, 2008.

[20] L. Abenavoli, N. Milic, M. Masarone, and M. Persico, "Association between non-alcoholic fatty liver disease, insulin resistance and Helicobacter pylori," Medical Hypotheses, vol. 81, no. 5, pp. 913-915, 2013. 


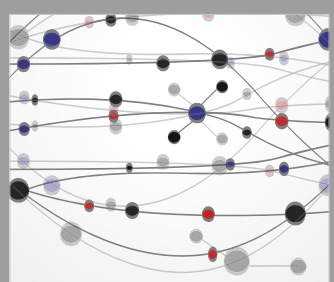

The Scientific World Journal
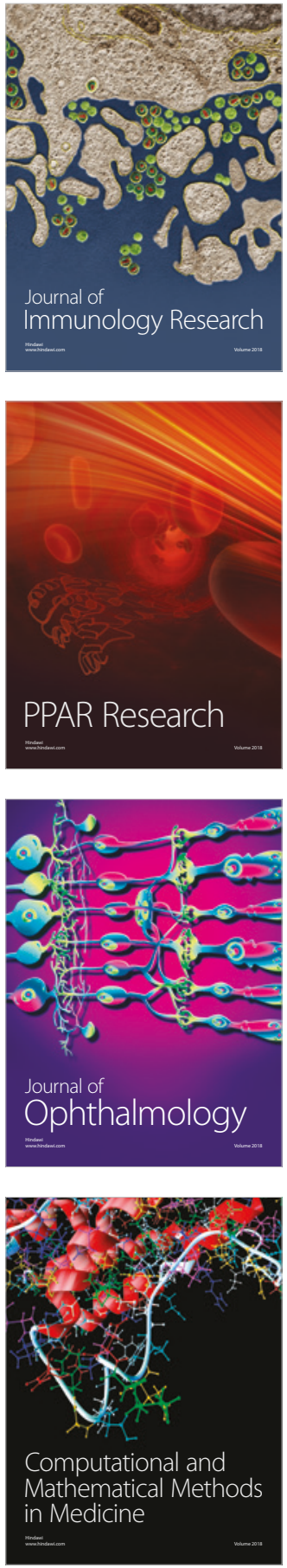

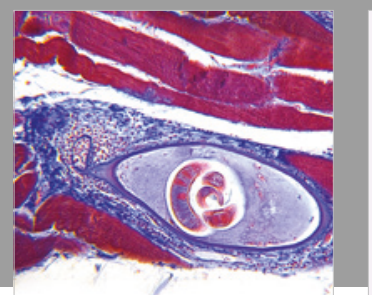

Gastroenterology Research and Practice

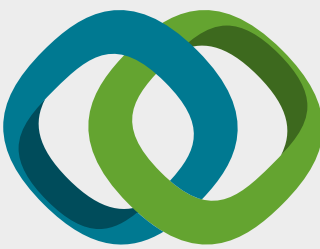

\section{Hindawi}

Submit your manuscripts at

www.hindawi.com
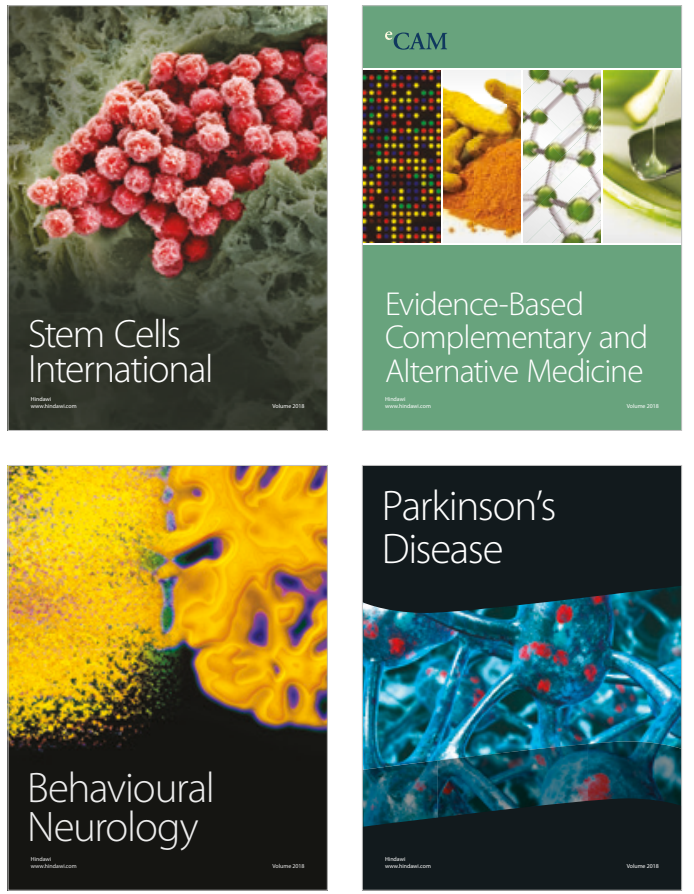

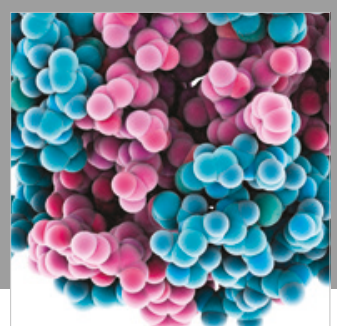

ournal of

Diabetes Research

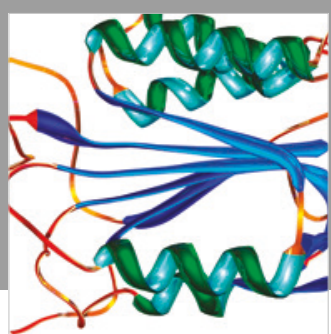

Disease Markers
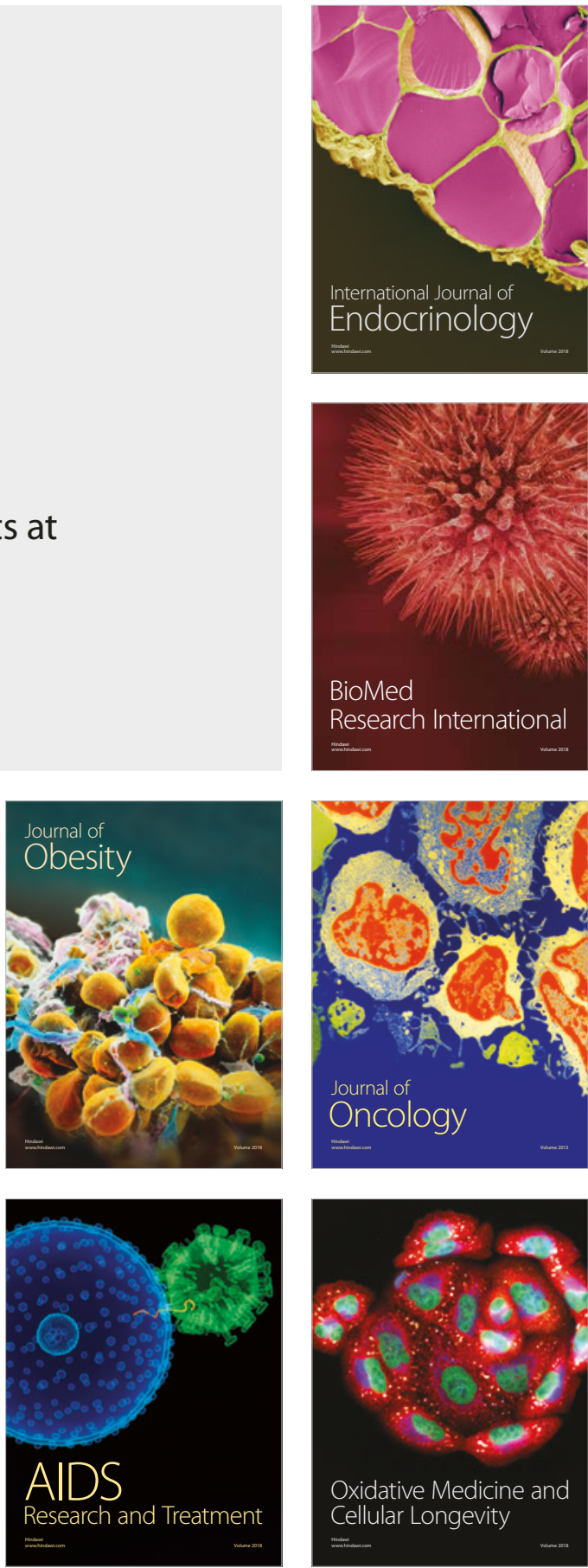